\title{
Towards A Pan-European Pension System: An Initial Approach
}

\author{
Panagiotis Kardiasmenos \\ Nikos Frangos \\ Department of Statistics \\ Athens University of Economics and Business \\ Patission Ave. 76, Athens 10434 \\ Greece
}

\begin{abstract}
In this work we propose a Pan-European pension System. We are in the process of evaluating our proposal actuarially.

Aiming to present a new practical first tier related Welfare Model for a Catholic European Pension, we take into account the rules that dictated the establishment and growth of the past welfare typologies. The creation of social welfare models on a theoretical level, concerning their distinction and function, begun primarily in 1958 through the work of Wilensky and Lebeaux. Twenty years later, the social sciences following the distinction that has occurred between the residual (or marginal) and institutional (or integrated) welfare, have had a fertile ground to develop the widely known as welfare benefits typologies.

The distinction between the existing welfare types and the creation of new ones, offers a determinant frame of reference on the Welfare State strengths and weaknesses. The purpose of the distinction was done in favor of the academic observation and, where it was deemed necessary, for pinpointing their qualities through the given proposals in the dissertation. However, it must be noted that typologies and models do pose certain considerable concerns.
\end{abstract}

First, as it has been aforementioned, the welfare model typology is not a satisfactory classification system. That happens because no actual welfare state encompasses all the decisive properties a model typology presents theoretically, but rather is an amalgamation of different welfare features.

Another important disadvantage, revealed through the analysis of the Euro-zone members, is that while ideal models are essentially static as concepts, the Member State Welfare is a on the contrary a dynamic process in which policies are constantly shifting.

In addition, the Welfare Models may be indeed useful for descriptive purposes, yet they provide no information on explaining observable or not phenomena, nor moreover predict the appearance or absence of those particular phenomena. That is why each Welfare Model is based on an ideal situation in time which limits their contextual analysis.

Considering the Welfare Model challenges, it is apparent that choosing a strict rulebook for generating and analyzing Welfare Models is considered to be a sensible tactic only when taking into account their limitations. For that reason, the most prevalent way to approach the Welfare Models nowadays is by examining their financial aspects, hence the route taken by the presented dissertation.

In conclusion it is therefore now apparent on why a divergence occurs in the Welfare Models examination, when several countries are included in the sample, while consequently a policy loses its framework's reference (Gordon 1988). However, in case of this dissertation, the laid out academic foundation and contribution is rigorously maintained on analyzing the subject by redefining the future potential of a unified Europe. 
Table X - Euro-zone Countries Information in Correlation to the First-Pillar Pension Benefits Data.

\begin{tabular}{|c|c|c|c|c|c|c|c|c|c|c|c|}
\hline $\begin{array}{c}\text { Member } \\
\text { State of the } \\
19\end{array}$ & $\begin{array}{c}\text { Pension } \\
\text { Age } \\
\text { Eligibility } \\
\text { Men }\end{array}$ & $\begin{array}{l}\text { Pension } \\
\text { Age } \\
\text { Eligibility } \\
\text { Women }\end{array}$ & $\begin{array}{l}\text { Minimum } \\
\text { Individual } \\
\text { Pension }\end{array}$ & $\begin{array}{l}\text { Country's } \\
\text { Population }\end{array}$ & $\begin{array}{c}\text { Income } \\
\text { Per Capita }\end{array}$ & $\begin{array}{c}\text { GDP } \\
\text { (Billion) }\end{array}$ & $\begin{array}{l}\text { Unemp } \\
\text { loyme } \\
\text { nt } \%\end{array}$ & $\begin{array}{c}\text { Life } \\
\text { Expectanc } \\
\text { y Women }\end{array}$ & $\begin{array}{c}\text { Life } \\
\text { Expectanc } \\
\text { y Men }\end{array}$ & $\begin{array}{l}\text { Pension } \\
\text { Expenditu } \\
\text { res On the } \\
\text { GDP }\end{array}$ & $\begin{array}{c}\text { Minimum } \\
\text { Years of } \\
\text { Employme } \\
\text { nt }\end{array}$ \\
\hline Austria & 65 & 60 & $€ 882.78$ & 8.823 .054 & $\$ 49.869$ & $\$ 439.6 \delta \mathrm{ic}$ & $12,5 \%$ & 84,3 & 81,5 & $14.6 \%$ & 40 \\
\hline Belgium & 65 & 65 & $€ 708.0$ & 11.358 .357 & $\$ 44.881$ & $\begin{array}{l}\$ 470.179 \\
\text { Billion }\end{array}$ & $14,5 \%$ & 83,4 & 78,7 & $12.7 \%$ & 41 \\
\hline France & $\begin{array}{c}61 \mathrm{y} \& 7 \mathrm{~m} \\
\text { or } 66 \mathrm{y} \& \\
7 \mathrm{~m}\end{array}$ & $\begin{array}{c}61 \mathrm{y} \& 7 \mathrm{~m} \\
\text { or } 66 \mathrm{y} \& \\
7 \mathrm{~m}\end{array}$ & $€ 379$ & 67.795 .000 & $\$ 38.127$ & $\begin{array}{l}\$ 2.463 \\
\text { Billion }\end{array}$ & $15,1 \%$ & 85,3 & 79,5 & $15 \%$ & 42 \\
\hline Germany & 65 y $4 / 5 \mathrm{~m}$ & 65 y $4 / 5 \mathrm{~m}$ & $\begin{array}{l}\text { No Max or } \\
\text { Minimum }\end{array}$ & 82.521 .653 & $\$ 44.500$ & $\begin{array}{l}\$ 3.685 \\
\text { Billion }\end{array}$ & $9,2 \%$ & 83,4 & 78,7 & $11.8 \%$ & 35 \\
\hline Greece & 67 & 67 & $€ 345.6-384$ & 10.816 .286 & $\$ 20.579$ & $\begin{array}{l}\$ 221,5 \\
\text { Billion }\end{array}$ & $23,9 \%$ & 83,5 & 78,4 & $17.8 \%$ & 15 \\
\hline Estonia & 63 & 63 & $\begin{array}{c}€ 409^{*} \\
\text { (Mean) }\end{array}$ & 1.318 .705 & $\$ 17.632$ & $\begin{array}{l}\$ 23,13 \\
\text { Billion }\end{array}$ & $10,1 \%$ & 81,9 & 73,1 & $8 \%$ & 15 \\
\hline Ireland & 66 & 66 & $€ 933.2$ & 4.761 .865 & $\$ 80.641$ & $\begin{array}{l}\$ 385 \\
\text { Billion }\end{array}$ & $14,1 \%$ & 83,6 & 78,2 & $5.5 \%$ & 10 \\
\hline Spain & 65 & 65 & $€ 636.1$ & 46.549 .000 & $\$ 26.608$ & $\begin{array}{l}\$ 1.232,59 \\
7 \text { Billion }\end{array}$ & $23,4 \%$ & 85,4 & 79,9 & $12.6 \%$ & 15 \\
\hline Italy & 66 & 66 & $€ 501.89$ & 60.507 .590 & $\$ 30.507$ & $\begin{array}{l}\$ 1.850,73 \\
7 \text { Billion }\end{array}$ & $14,3 \%$ & 84,9 & 80,5 & $16.5 \%$ & 20 \\
\hline Cyprus & 65 & 65 & $€ 336.2$ & 854.800 & $\$ 23.351$ & $\begin{array}{c}\$ 19,810 \\
\text { Billion }\end{array}$ & $13,2 \%$ & 83,1 & 78,4 & $10.8 \%$ & 13 \\
\hline Latvia & $63 y \& 3 m$ & 63 y $3 m$ & $\begin{array}{c}€ 200 \\
\text { (Estimation) }\end{array}$ & 1.929 .900 & $\$ 30.507$ & $\begin{array}{l}\$ 14.060 \\
\text { Billion }\end{array}$ & $18,3 \%$ & 79,6 & 70 & $7.7 \%$ & 15 \\
\hline Lithuania & 63 & 61 & $€ 288$ & 2.810 .865 & $\$ 19.534$ & $\begin{array}{c}\$ 54,352 \\
\text { Billion }\end{array}$ & $21,4 \%$ & 80,2 & 69,7 & $6.8 \%$ & $\begin{array}{c}15 \\
\text { (Since } \\
201830 \\
\text { Years) }\end{array}$ \\
\hline Luxemburg & 65 & 65 & $€ 890$ & 602.005 & $\$ 103.198$ & $\begin{array}{c}\$ 59,468 \\
\text { Billion }\end{array}$ & $6,4 \%$ & 84,6 & 80,7 & $9.3 \%$ & 40 \\
\hline Malta & 60 & 60 & $€ 431$ & 434.403 & $\$ 25.214$ & $\begin{array}{c}\$ 10,953 \\
\text { Billion }\end{array}$ & $5,0 \%$ & 83,3 & 79,6 & $7.6 \%$ & 35 \\
\hline $\begin{array}{l}\text { Netherland } \\
\text { s }\end{array}$ & 65 & 65 & $€ 1,113.56$ & 17.188 .222 & $\$ 45.282$ & $\begin{array}{l}\$ 771,163 \\
\text { Billion }\end{array}$ & $6,6 \%$ & 83,2 & 80 & $13 \%$ & $\begin{array}{l}\text { There is a } \\
\text { different } \\
\text { function } \\
\text { after the } \\
55 \text { years }\end{array}$ \\
\hline Portugal & 66 & 66 & $€ 201.53$ & 10.379 .573 & $\$ 24.237$ & $\begin{array}{c}\$ 248,891 \\
\text { Million }\end{array}$ & $9,3 \%$ & 84,5 & 78,3 & $14.9 \%$ & 15 \\
\hline Slovakia & 62 & 62 & $€ 337.38$ & 5.435 .343 & $\$ 20.508$ & $\begin{array}{c}\$ 111 \\
\text { Million }\end{array}$ & $27,4 \%$ & 80,9 & 73,8 & $8.6 \%$ & 30 \\
\hline Slovenia & 68 & 68 & $€ 181.36$ & 2.066 .880 & $\$ 27.535$ & $\begin{array}{c}\$ 56,933 \\
\text { million }\end{array}$ & $10,5 \%$ & 83,7 & 78 & $10.9 \%$ & 15 \\
\hline Finland & 65 & 65 & $€ 636.63$ & 5.509 .717 & $\$ 52.422$ & $\begin{array}{l}\$ 289,557 \\
\text { Billion }\end{array}$ & $10,9 \%$ & 80,9 & 73,8 & $13.1 \%$ & $\begin{array}{l}\text { Guarantee } \\
\text { d Pension }\end{array}$ \\
\hline $\begin{array}{l}\text { Totals \& } \\
\text { Mediums }\end{array}$ & 64,7 & 64,3 & - & $\begin{array}{c}341.008 .86 \\
7\end{array}$ & $\$ 40.999$ & $\begin{array}{l}12.589,50 \\
\text { Billion }\end{array}$ & $13 \%$ & 78,9 & 77,4 & - & - \\
\hline
\end{tabular}

Source: OECD - Pension at Glance $2017^{1}$, EUROSTAT ${ }^{2}$ 
According to Table (X) the parameters of a new supranational pension model would include the already established and satisfactorily retirement age limit of 65 years, which could be applied as universal retirement age. Nonetheless it should be underlined that according to the theory section of the dissertation, many countries in Europe are planning to increase the retirement age limit at the age of 67 years within the next decade, due to the rapidly aging population.

The mean pension in that case would be determined according to the replacement rate in each country as shown in Table (X2). Yet in order for the mean pension to be beneficiary the average per capita income in the Euro-zone should be $\$ 40.990$. That per capita income could be achieved through initiatives in education and employment which would then allow the revenue growth to rise and that would justify an increase in the total retirement income of the Euro-zone.

Consequently, if a constructed Welfare Model achieves full retirement at 66 and a life expectancy of about 80 years it will also be able to predict the fifteen-year pension provision of each generation. On the contrary, an issue arises in the case of the minimum working years for retirement, where there are many variables that cannot always be measured and as a result be controlled for future pension provisions to be predictable.

Table (X2) - 2017 Pension Replacement Rates by OECD.

\begin{tabular}{|c|c|c|c|}
\hline Euro-zone Member State & $\begin{array}{c}\text { Pension Replacement Rate \% } \\
0.5 \text { (Low) }\end{array}$ & $\begin{array}{c}\text { Pension Replacement Rate \% } \\
\text { (Medium) }\end{array}$ & $\begin{array}{c}\text { Pension Replacement Rate \% } \\
1.5 \text { (High) }\end{array}$ \\
\hline Austria & 92.2 & 91.8 & 90.9 \\
\hline Belgium & 62.6 & 66.1 & 50.1 \\
\hline France & 70.4 & 74.5 & 70.3 \\
\hline Germany & 54.7 & 50.5 & 49.8 \\
\hline Greece & 60.7 & 53.7 & 54.1 \\
\hline Estonia & 73.7 & 57.4 & 51.1 \\
\hline Ireland & 70 & 42.3 & 32.4 \\
\hline Spain & 79.3 & 81.8 & 81.7 \\
\hline Italy & 93 & 93.2 & 98.8 \\
\hline \multicolumn{4}{|l|}{ Cyprus* } \\
\hline Latvia & 55.7 & 59.5 & 59 \\
\hline \multicolumn{4}{|l|}{ Lithuania* } \\
\hline Luxemburg & 98.3 & 88.4 & 83.6 \\
\hline \multicolumn{4}{|l|}{ Malta* } \\
\hline Netherlands & 105.1 & 100.6 & 100.2 \\
\hline Portugal & 92.9 & 94.9 & 93.1 \\
\hline Slovakia & 85 & 83.8 & 83.5 \\
\hline Slovenia & 57.3 & 59.2 & 56.6 \\
\hline Finland* & & & \\
\hline
\end{tabular}

\footnotetext{
${ }^{2}$ https://ec.europa.eu/eurostat/statistics-explained/index.php/Social_protection_statistics_pension_expenditure_and_pension_beneficiaries 
Finally, the replacement rate on the pensions in the Eurozone, according to the European Community and the available Eurostat data, is 58\%. That percentage remains unchanged in the European Union of the 28 members.

Alternatively, a minimum Pan-European pension could be established i.e. $700 €$ per month. This pension could be finished by a Pan-European tax levy. This amount will be subtracted from the national pension. This way the old age problem will be distributed among all EU countries and at the same time will make workers mobility much easier. These two approaches are evaluated Actuarially in the current Dissertation.

* They do not exist in the OECDs assessment and therefore cannot be added for comparison in this table. The only Replacement rates which are missing are the countries that are not included in the OECD reports.

\section{Bibliography}

Gordon, M. S. (1988). Social Security Policies in Industrialized Countries. Cambridge: Cambridge Press. Wilensky, H. \& Lebeaux, C. N. (1958). Industrial Society and Social Welfare. New York: The Free Press.

\section{Typologies:}

Austria

https://www.demogr.mpg.de/papers/working/wp-2012-026.pdf

https://editorialexpress.com/cgi-bin/conference/download.cgi?db_name=SAEe2012\&paper_id=241

Belgium

https://www.plan.be/admin/uploaded/201005110837500.wp201010.pdf

France \& Germany

Robert Holzmann_Edward Palmer - Pension Reform_ Issues and Prospect for Non-financial Defined Contribution (NDC) Schemes (Trade and Development) (2006) (Book included in the email)

Lithuania and Esthonia

https://www.tandfonline.com/doi/pdf/10.1080/1406099X.2000.10840371

Latvia

http://dspace.lu.lv/dspace/bitstream/handle/7/31853/298-53144-Rajevska_Olga_or12003.pdf?sequence=1

Spain

Coping with Spain's Aging: Retirement Rules and Incentives

https://books.google.gr/books?id=SbH-

IhdXIGMC\&pg=PA9\&lpg=PA9\&dq=pensions $+\sigma p a i n+$ equation + formula\&source=bl\&ots=piGYo5BBiY\&sig=nc3XMYxaB81UBmfruuD U0dEpIQ4\&hl=en\&sa=X\&ved=2ahUKEwjAz53ooMLfAhWIa1AKHbU2BCoQ6AEwAnoECAcQAQ\#v=onepage\&q=pensions\%20 $\sigma p a i n$ $\% 20$ equation $\% 20$ formula\&f $=$ false

Italy

http://www.cerp.carloalberto.org/wp-content/uploads/2008/12/ageing_italy_fiche_en.pdf

Cyprus

https://books.google.gr/books?id=UDIi8-

qDSOIC\&pg=PA46\&lpg=PA46\&dq=pensions+cyprus+equation+formula\&source=bl\&ots=XMh_p6ZUez\&sig=1-

6UARdaGiRuRi3Bo74mVJYnr-

Q\&hl=en\&sa=X\&ved=2ahUKEwi429WwocLfAhVNZFAKHcmQDwAQ6AEwC3oECAMQAQ\#v=onepage\&q=pensions\%20cyprus\%20 equation $\% 20$ formula $\& \mathrm{f}=$ false

Netherlands

https://core.ac.uk/download/pdf/6670709.pdf

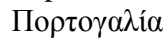

https://10envolver.files.wordpress.com/2012/05/pedro-rodrigues-phd-thesis-final-dec-21-2006.pdf

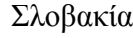

https://ec.europa.eu/info/sites/info/files/economy-finance/final_country_fiche_sk.pdf

http://www.ineko.sk/reformy2003/menu_dochodky_kalkulacka_paper2.pdf

Finland

https://www.julkari.fi/bitstream/handle/10024/129129/OptimisingpensionfinancingwithanapplicationtotheFinnishearningsrelatedpensionsc heme.pdf?sequence=1

Not Available

Slovenia

Irland

Luxemburg

Malta 